\title{
Editorial: What Level of Added or Free Sugar Is Commensurate With Good Health Outcomes?
}

\author{
Jennie Brand-Miller* \\ School of Life and Environmental Sciences and Charles Perkins Centre, The University of Sydney, Sydney, NSW, Australia
}

Keywords: total sugars, added sugars, free sugars, sugar-sweetened beverages, chronic disease, artificial sweeteners

Editorial: on the Research Topic

What Level of Added or Free Sugar Is Commensurate with Good Health Outcomes?

Is there a sweet spot for added sugars? There is consensus that any source of excess calories will contribute to weight gain and metabolic disease, but there is still debate on the level of added or free sugars which is commensurate with both good health and enjoyment of food. While guidelines for the range of energy (\%E) as carbohydrate, fat, and protein have widened, the reverse is true of added sugar. In previous decades, health authorities agreed that $10 \% \mathrm{E}$ was an appropriate upper cut-off, even if strong evidence was lacking. Nonetheless, since 2015, there have been moves to reduce that cut-point to $5 \% \mathrm{E}(1,2)$. In the eyes of many, eating as little sugar as possible is ideal.

I have concerns about limiting added (or free sugars) to $<5 \% \mathrm{E}$. This perspective is informed by knowledge of food science and technology, human evolution, and the role that sweetness plays in encouraging the consumption of healthy foods (e.g., wholegrains). The paradox of falling consumption of added sugars with increasing prevalence of overweight and obesity is now evident in many developed countries $(3,4)$. In Australia, peak intake of micronutrients is observed within the range $5-15 \% \mathrm{E}$ from free sugars (5). But of greater concern is the potential of unanticipated and undesirable consequences of health advice on added sugars and sugar-sweetened beverages (SSB). These include the increased incidence of restrictive eating disorders such as orthorexia nervosa (6) and an increase in alcohol consumption and deaths due to alcohol-related disease (7). In Australia, the consumers who avoid SSB, drink twice as many calories in the form of alcoholic beverages as the highest consumers of SSB (8). We should also recall that the history of nutrition science is replete with examples of where we got it wrong, including the "great protein fiasco" (9) and low-fat diets (10).

In this special issue of Frontiers in Nutrition, we hoped that the "sweet spot" (the highest level associated with no effect or harm) could be defined with a greater level of certainty. In a welldesigned 4-week randomized controlled trial conducted by Te Morenga et al., overweight adults ( $n=48$ ) randomized to consuming $1,800 \mathrm{~kJ}$ of SSB $(\sim 100 \mathrm{~g}$ added sugar, equivalent to $\sim 1,000 \mathrm{~mL}$ of SSB or $\sim 20 \% \mathrm{E}$ ) showed no changes in weight, blood pressure or other cardiometabolic factors compared with those assigned to consuming fruit with a similar energy content $(97 \mathrm{~g}$ naturallyoccurring sugars). However, men (but not women) showed an increase in uricemia, a risk factor for gout. Clearly, further studies in vulnerable groups of similar design and longer duration are needed.

Some studies directly addressed the question of safe levels of intake. In a Swedish population $(n=22,877)$, during a mean follow-up of nearly 20 years, $>20 \% \mathrm{E}$ as added sugar was associated with increased coronary events $(\mathrm{HR}=1.39)$ and stroke risk $(\mathrm{HR}=1.31)$ compared to $7.5-10 \% \mathrm{E}$ as added sugar (Janzi et al.). Surprisingly, participants with the lowest intake $(<5 \% \mathrm{E})$ had the highest risk of atrial fibrillation and aortic stenosis. This result is difficult to explain but emphasizes 
the complexity of nutrition and observational studies. In a wellcharacterized older cohort of Australians $(n=1,713)$, changes in the $\% \mathrm{E}$ from added sugars were not associated over time with changes in body weight, regardless of source-beverage or nonbeverage (Moshtaghian et al.). Earlier work in this cohort found those consuming $<5 \% \mathrm{E}$ as added sugar, were higher consumers of alcohol (11).

Added sugars are used in tiny amounts $(\sim 1-2 \% \mathrm{w} / \mathrm{w})$ to enhance flavors and improve palatability. If this encourages excessive energy intake, then the same must be said of salt, herbs, spices, and soy sauce, all of them in use for thousands of years. In large amounts, the physicochemical, technological, and functional characteristics of sugars influence human metabolism as reviewed by Brouns. Sugars also influence dental health. The effect of sugars on oral health was the conditional reason that $\mathrm{WHO}$ recommended consumption of free sugars to below $5 \%$ of total energy (2). But Brouns reminds us that frequency, contact time, and rapidly digestible starches and acidic foods like wine and fruit also affect dental health. Reducing the amount of added sugars from SSB will not be effective if starchy snacks and sticky confectionery are consumed instead (3).

Determining the extent to which added sugars contribute to disease in various populations is challenging because it is difficult to accurately measure intakes. Biomarkers of sugar intake may therefore be helpful although this does not distinguish added sugars from sugars in fruit and vegetables. Te Morenga's second paper (Te Morenga et al.) found that the sum of urinary [sucrose + fructose] was weakly but significantly correlated $(\mathrm{r}=$ 0.23 ) with intakes of total sugars and with added sugars from SSB $(n=0.26)$. Interestingly, they found a higher correlation $(\mathrm{r}=0.40)$ with the $\mathrm{C}-13$ carbon isotope ratio of alanine. Similarly, in the DONALD study ( $n=254$ adolescents), Della Della Corte et al. backed up dietary records with measurement of fructose and the sum of [fructose + sucrose] in two complete 24-h urine collections. They found no prospective associations between adolescent intake of fructose, sucrose, glucose, added, free, and total sugar with adult insulin sensitivity as measured by HOMA2-\%S. Indeed, higher fructose in urine was associated with improved insulin sensitivity in females (but not males).

An underlying assumption of recommendations to reduce SSBs, is that water will take their place. In the US population (NHANES, $n=22,716$ ), Drewnoski's group reported that SSB consumption had declined by $\sim 20 \%$ in volume between 2011 and

\section{REFERENCES}

1. Scientific Advisory Committee on Nutrition. Carbohydrates and Health. London: The Stationery Office Ltd (2015).

2. World Health Organisation. Guideline: Sugars Intake for Adults and Children. Geneva: World Health Organisation (2015).

3. Brand-Miller JC, Barclay AW. Declining consumption of added sugars and sugar-sweetened beverages in Australia: a challenge for obesity prevention. Am J Clin Nutr. (2017) 105:854-63. doi: 10.3945/ajcn.116.145318
2016 (Vieux et al.), whereas plain and bottled water increased by just $\sim 10 \%$. The opposing time trends were not uniform-lower income and minority groups consumed more bottled water and relatively little tap water. In this context, changes in intake of alcoholic beverages and other sources of energy (e.g., chocolate) must be explored.

Pang et al. attracted the highest number of views with their review of the current state of knowledge on artificial sweeteners, reminding us that they are not all the same, with different chemical structures, absorption, and metabolic effects. Despite many being in use for 50 years, there are still very few longterm studies to show that substituting sugars and SSB with non-caloric alternatives is of benefit. And finally, an updated meta-analysis and systematic review by Zafar et al. confirmed that chronic consumption of fructose is neither more beneficial nor harmful than sucrose or glucose for glycemia and other metabolic outcomes.

Taken together, this collection of 11 papers provides evidence that a diet containing $>20 \%$ added sugars may have adverse effects, but so too, a diet containing $<5 \%$ added sugar. At worst, such a restrictive diet can create food fear or an unhealthy relationship with food and alcohol, especially for women and girls. As the Swedish study found (Janzi et al.), the sweet spot may therefore lie somewhere between 7.5 and $10 \% \mathrm{E}$ as added sugars. Many will agree that public health interventions and food taxes to prevent obesity and related diseases should promote the quality of the overall diet, not a singular focus on reducing sugar and SSB intakes.

\section{AUTHOR CONTRIBUTIONS}

The author confirms being the sole contributor of this work and has approved it for publication.

\section{FUNDING}

JB-M is a recipient of past and current National Health and Medical Research funding from the government of Australia.

\section{ACKNOWLEDGMENTS}

I thank Ted Kyle and Alan Barclay for helpful feedback on the Editorial and my co-editor Professor Anette Buyken and all the peer reviewers who took time to review the papers for this special issue.
4. Rippe JM, Angelopoulos TJ. Added sugars and risk factors for obesity, diabetes and heart disease. Int J Obesity. (2016) 40:S22-7. doi: 10.1038/ijo.20 16.10

5. Mok A, Ahmad R, Rangan A, Louie JCY. Intake of free sugars and micronutrient dilution in Australian adults. Am J Clin Nutr. (2018) 107:94-104. doi: 10.1093/ajcn/nq $\mathrm{x} 008$

6. Zickgraf HF, Ellis JM, Essayli JH. Disentangling orthorexia nervosa from healthy eating and other eating disorder symptoms: relationships with clinical impairment, comorbidity, and self-reported food 
choices. Appetite. (2019) 134:40-9. doi: 10.1016/j.appet.2018.1 2.006

7. Chikritzhs T, Allsop SJ, Moodie AR, Hall WD. Per capita alcohol consumption in Australia: will the real trend please step forward? Med J Austral. (2010) 193:594-7. doi: 10.5694/j.1326-5377.2010.tb0 4069.x

8. Wong THT, Buyken AE, Brand-Miller JC, Louie JCY. Is there a soft drink vs. alcohol seesaw? A cross-sectional analysis of dietary data in the Australian Health Survey 2011-12. Euro J Nutr. (2020) 59:235767. doi: 10.1007/s00394-019-02084-4

9. Mclaren D. The great protein fiasco. Lancet. (1974) 304:936. doi: 10.1016/S0140-6736(74)91649-3

10. Ludwig DS. Lowering the bar on the low-fat diet. JAMA. (2016) 316:20878. doi: 10.1001/jama.2016.15473

11. Moshtaghian H, Louie JCY, Charlton KE, Probst YC, Gopinath B, Mitchell $\mathrm{P}$, et al. Added sugar intake that exceeds current recommendations is associated with nutrient dilution in older Australians. Nutrition. (2016) 32:937-42. doi: 10.1016/j.nut.2016.02.004
Conflict of Interest: JB-M is President of the Glycemic Index Foundation and overseas a glycemic index testing service at the University of Sydney. She receives royalties from the University of Sydney and popular books about nutrition and health.

Publisher's Note: All claims expressed in this article are solely those of the authors and do not necessarily represent those of their affiliated organizations, or those of the publisher, the editors and the reviewers. Any product that may be evaluated in this article, or claim that may be made by its manufacturer, is not guaranteed or endorsed by the publisher.

Copyright (C) 2021 Brand-Miller. This is an open-access article distributed under the terms of the Creative Commons Attribution License (CC BY). The use, distribution or reproduction in other forums is permitted, provided the original author(s) and the copyright owner(s) are credited and that the original publication in this journal is cited, in accordance with accepted academic practice. No use, distribution or reproduction is permitted which does not comply with these terms. 